\title{
Sleep Macroarchitecture in Depression: Sex Differences
}

\author{
Leslie M. Swanson*, Robert Hoffmann and Roseanne Armitage
}

Department of Psychiatry University of Michigan, USA

\begin{abstract}
Background: Although the relationship between sleep and depression has been studied over the past two decades, findings are inconsistent across the literature. The present study evaluated differences in sleep macroarchitecture between adults with major depressive disorder (MDD) and healthy controls, paying particular attention to sex differences.

Method: Sleep macroarchitecture was investigated in 129 adults symptomatic with MDD compared to 62 never-depressed healthy controls. Participants were free of psychiatric medications and were required to maintain a set sleep schedule for the 5 days prior to study.

Results: Prolonged sleep latency and increased REM density were observed in participants with MDD relative to healthy controls, but REM latency was longer and there were fewer arousals in the MDD group. Regression analysis differentiated between MDD and HC groups, but only in men. Sex differences were also found in the relationship between clinical course of MDD and sleep variables with stronger correlations between sleep, age of onset and MDD severity in men than in women.

Conclusions: Sex differences were observed in the relationship between clinical course of depression and sleep and in differentiating patients form healthy controls, supporting a different pathophysiology of depression in men and women.
\end{abstract}

Keywords: Sleep, depression, sex differences, healthy controls, EEG.

\section{INTRODUCTION}

Major depressive disorder (MDD) is the most common psychiatric illness, affecting nearly 14 million adults each year. MDD is the leading cause of disability for Americans between 15-44 years of age, and is associated with suicide, decreased quality of life, and impaired functioning [1-6]. Sleep disturbance is a frequently observed symptom of MDD [7], and is implicated in the etiology and clinical course of depression. Insomnia and sleep disturbance independently increase risk for the development, relapse, or recurrence of a major depressive episode, and are associated with increased risk of suicide [8-16].

The connection between sleep and depression has been the subject of numerous studies over the past 20 years. Electroencephalographic (EEG) sleep alterations are thought to be biological markers for depression and multiple studies have examined EEG differences in individuals with depression versus healthy controls. However, many findings are discrepant between studies, and unanswered questions remain. Methodological issues, such as age and sex confounds, small sample sizes, and poorly controlled participant variables (e.g., irregular sleep schedules, unmonitored caffeine, alcohol, or drug use), are major contributors to these discrepancies. We sought to address these methodological issues in the present study and to update the body of work on depression and sleep macroarchitecture, based on visual scoring of sleep polysomnography.

*Address correspondence to this author at the Department of Psychiatry, University of Michigan, 4250 Plymouth Rd, Ann Arbor, MI, 48109-5763, USA; Tel: 7347642242; Fax: 7347641229;

E-mail: LMSwan@med.umich.edu
Various differences in sleep macroarchitecture between individuals with depression and healthy controls have emerged from pervious research. Specifically, sleep continuity problems (prolonged sleep latency [17-19], increased wakefulness during the night [20], decreased sleep efficiency $[18,20])$, slow wave abnormalities $[19,21]$, and decreased REM latency [20-22] have been observed in participants with MDD in laboratory studies, although it is important to note that these findings are not consistent across the literature $[23,24]$. Furthermore, many of the differences found between healthy controls and individuals with depression are evident only in studies that use older samples [23]. To date, no single sleep variable has been found to reliably distinguish individuals with depression from healthy controls [25]. Notably, the majority of research on sleep macroarchitecture has neglected variables related to sleep stability and arousal, such as ascents to stage 1 sleep and sleep stage changes per hour. Thus, examination of these variables represents an opportunity to add to the existing body of literature on sleep macroarchitecutre and depression.

Despite the sizable number of studies examining EEG differences in healthy controls versus depressed individuals, very few recent studies have examined sex differences in sleep variables between participants with depression and healthy controls. Although there is compelling evidence that depression pathophysiology is different between men and women [24], the literature regarding sex differences in sleep variables remains equivocal and controversial, with several studies finding few differences between males and females with MDD [26, 27], while others report robust EEG differences [28-30]. Among those studies detecting sex differences in MDD, the most robust finding is that men with MDD show lower amplitude slow-wave activity (SWA), also 
known as delta activity in NREM sleep. The time course of SWA and response to homeostatic sleep challenge is also blunted in men with MDD [29-31]. By contrast, women with depression do not show reduced SWA and may be hyperresponsive to sleep challenge [30, 31]. Further, MDD women are also more likely to show poorly synchronized sleep EEG rhythms, and increased fast-frequency EEG activity during sleep $[30,32]$. However, not all studies include EEG frequency analysis and sleep macroarchitecture remains the "gold standard" of research in this area.

The goal of the present study was to further elucidate the relationship between sleep macroarchitecture and depression using a methodology that addresses many of the shortcomings of the existing literature, including use of a large, agematched sample of adults with depression and healthy controls studied under carefully controlled laboratory conditions as described below. We also controlled for the influence of age in our analyses and directly examined sex differences between groups and within the MDD group.

\section{MATERIALS AND METHODOLOGY}

\section{Participants}

Data for this study were obtained from an archival database, collected under identical conditions over the past decade, including approximately half of the subjects recorded in the University of Texas Southwestern Medical Center Sleep Study Unit (UTSW) and the remaining portion of the sample collected in the University of Michigan Sleep and Chronophysiology Laboratory, when our research group relocated to Michigan. Participants were recruited from a number of sources. Participants were recruited from the community using advertisements, word of mouth, and flyers posted in public places or by physician referral for patients.

Written informed consent was obtained from all subjects under protocols approved by the Institutional Review Board (IRB) of either UTSW or the University of Michigan (UM). A waiver of consent was approved by the UM IRB to analyze the archival data collected at UTSW.

All participants underwent a full Structured Clinical Interview for the DSM-III-R [33] or DSM-IV, which allows the interviewer to determine whether the participant currently meets, or has ever met, diagnostic criteria for psychiatric disorders. Personal and family psychiatric and medical history was obtained. Depressive symptom severity was assessed using the 17-item Hamilton Rating Scale for Depression (HRS-D [34]), a clinician-rated depression severity scale. See Table 1 for selected characteristics of the sample.

\section{Depressed Outpatients}

One hundred and twenty-nine outpatients (41 men and 88 women) 18 to 67 years old (mean $36 \pm 11$ years), who met DSM-III-R [33] or DSM-IV [7] criteria for current major depressive disorder and had moderate symptom severity greater (> 17) on the 17-item Hamilton Rating Scale for Depression (HRS-D [34]) were included in the study. The average age for men was $35 \pm 11$ years, and for women $36 \pm 11$ years. Exclusion criteria included comorbid Axis I disorders, use of psychotropic medications in past eight weeks, other medical conditions, or substance abuse within the past 12 months.

\section{Never-Depressed Healthy Controls}

Sixty-two healthy controls (HC) 20 to 64 years old (mean $29 \pm 8$ years) also participated in the study. Thirty-two men and 30 women were included. The average age was $28 \pm 6$ years for men and $31 \pm 10$ years for the women. All healthy controls were medically and psychiatrically healthy, and had no lifetime personal or family history of depression, other Axis I disorders, or substance abuse, based on the SCID for DSM-III-R or DSM-IV. All controls completed the HRS-D to verify that they were not experiencing any symptoms of depression at the time of study (all had HRS-D scores < 3). Therefore, all healthy controls had never experienced a depressive episode, had no family history of depression, and were not currently experiencing any symptoms of depression.

In addition, inclusion in the data analysis required that participants had no protocol violations and two consecutive nights of sleep data with no technical recording problems, and no missing data. Participants were screened for evidence of independent sleep disorders (e.g., narcolepsy, bruxism, restless legs syndrome, periodic limb movement disorder, apnea) using clinical history and polysomnography on the first night in the laboratory. Participants who were shift workers or had signs or symptoms of independent sleep disorders were excluded. Participants were required to maintain regular bed and rise times for 5 days prior to study, confirmed by actigraphy and self-report sleep diaries. Their habitual sleep schedule was also followed in the laboratory on nights of study.

Participants were adapted to the laboratory setting by completing an extensive tour of the sleep environment with the research assistant who would conduct their sleep study. All participants were instructed to refrain from napping, consuming alcohol or illegal drugs, consuming caffeine after late morning, and to minimize their caffeine consumption, for five days prior to study. Breathalyzer testing and urine drug screens provided confirmation that participants followed protocol regarding drug and alcohol use. All participants were medication free at the time of study. Those previously treated with antidepressant medications had been discontinued 4-8 weeks prior to study, for reasons other than the purpose of the study. Females who were not using oral or implant contraceptives were studied during the early follicular phase of their menstrual cycle.

Participants slept for two consecutive nights in the lab at either the UTSW or the UM. Both laboratories were designed and run under the direction of two senior researchers (RA \& RH), with identical procedures and equipment. The first night served as an adaptation night and participants for additional screening $\mathrm{A}$ full electrode montage, including leg leads, chest and abdomen respiration bands, and nasal-oral thermistor, was used during the first night in the study. On the second night, the electrode montage included left (C3) and right central (C4) EEG, left and right EOG, recorded from upper and lower canthi, and a bipolar, chin-cheek EMG. EEG electrodes were referenced to the ear lobes linked together through to a 10 kilo-ohm resistor to minimize nonhomogeneous current flow and potential artifactual hemispheric asymmetries as is standard in our laboratory. EEG was transduced by GRASS P511 A/C amplifiers set to a sensitivity of $5(50 \mu \mathrm{V}, 0.5 \mathrm{~s}$ calibration) corresponding to a 
gain of 50,000. The half-amp low- and high-bandpass filters were set at 0.3 and $30 \mathrm{~Hz}$, respectively. A $60-\mathrm{Hz}$ notch filter attenuated electrical noise.

Table 1. Means and Standard Deviations (in brackets) of Selected Demographic and Clinical Characteristics of Participants

\begin{tabular}{|l|c|c|}
\hline & $\begin{array}{c}\text { MDD } \\
\mathrm{n}=129\end{array}$ & $\begin{array}{c}\mathrm{HC} \\
\mathrm{n}=62\end{array}$ \\
\hline \hline Males, $n(\%)$ & $41(32 \%)$ & $32(52 \%)$ \\
\hline Females, $n(\%)$ & $88(68 \%)$ & $30(48 \%)$ \\
\hline Age of males, years & $36(11)$ & $28(6)$ \\
\hline Age of females, years & $36(11)$ & $31(10)$ \\
\hline Age, years & $36(11)$ & $29(8)$ \\
\hline HRSD & $22.7(5.6)$ & N/A \\
\hline Age of MDD Onset & $25(11)$ & N/A \\
\hline \# Previous MDD Episodes & $3(2)$ & N/A \\
\hline
\end{tabular}

HRSD $=$ Hamilton Rating Scale for Depression, MDD = Sample with Major Depressive Disorder, $\mathrm{HC}=$ Healthy Control Sample.

Clinical characteristics (HRSD, age of MDD onset, and number of previous MDD episodes) are not applicable for the healthy control sample.

Signals were digitized on-line at $250 \mathrm{~Hz}(62.5 \mathrm{~Hz}$ for EOG and EMG) through a 16-bit MICROSTAR analogueto-digital converter and displayed on a digital polygraph system designed and validated in-house. Raw digitized data were stored on CD.

Sleep records were scored visually according to standard criteria [35] by research personnel trained at $>90 \%$ stage agreement on an epoch-by-epoch basis. All records were inspected visually and epochs containing artifact or recording difficulties were excluded from analysis. Several sleep variables were derived from the scored polysomnographic (PSG) data. See Armitage et al. [36] for computational detail. Briefly, persistent sleep latency (first 10 minutes of any stage with no more than 2 minutes of awake), the total sleep period (from sleep onset to wake-up time) sleep efficiency (the ratio of total sleep period to time in bed $x$ 100) REM latency (with no minimum duration criterion) and the percentages of each of stages 1-4, REM and awake were computed relative to the total sleep period. Awake and movement time was also computed in each third of the night. The durations of each complete REM period were also computed.

The data were coded for diagnostic group (MDD versus HC), sex, and age. The first step in data analysis was to conduct simple descriptive statistics. A series of regression analyses were chosen as the main analytic strategy to best capture the multivariate nature of the relationship between sleep and depression, informed by Benca et al.'s [25] work, which has suggested that a combination of sleep variables best discriminate between individuals with depression and healthy controls.

Given the plethora of PSG variables in the dataset, the first analysis used stepwise linear regression to determine which combination of sleep variables differed between patients with MDD and HCs, Only those variables accounting for a significant and unique proportion of the variance in discriminating between MDD and HC groups were retained, in an effort to reduce multicollinearity and increase statistical power [37]. Also note that the influence of chronological age was removed from all regression analyses, equivalent to treating age as a statistical covariate in an analysis of variance model.

To define the subset of variables that maximally discriminated participants with MDD from healthy controls a second stepwise regression analysis was performed using the retained PSG variables, using age as a statistical covariate.

\section{RESULTS \\ PSG Predictors of MDD}

The variables that did not meet inclusion criteria for the first stepwise regression were: Sleep Efficiency, \% Stage 1 sleep, \% Stage 2 sleep, \% Slow-Wave sleep, \% REM, \% Awake and movement, ascents to Stage 1 per hour, Stage changes per hour, and Time in bed. Of the remaining sleep variables, sleep latency, REM density, REM latency, and arousals in the last 150 minutes of sleep together significantly differentiated the MDD from HC groups. The overall regression accounted for $13 \%$ of the variance in group membership $(\mathrm{p}<.001)$. Specifically, as shown in Table 2 , participants with MDD took significantly longer to fall asleep and showed increased REM density compared to HCs, consistent with previous reports. However, the MDD group also had fewer arousals in the last 150 minutes of sleep and had longer REM latency than HCs. None of the remaining sleep measures added to the discrimination between groups.

\section{Sex Differences in Differentiating MDD from HC Groups}

The second set of stepwise regression analyses compared PSG predictors of disease status separately for males and females. Among males, four predictors (sleep efficiency, ascents to Stage one per hour, stage changes per hour, and REM density) accounted for $26 \%$ of the variance in MDD status $(\mathrm{p}<.001)$. Compared to HCs , males with MDD had decreased sleep efficiency, increased REM density, fewer ascents to Stage one, as well as fewer stage changes per hour of sleep. By contrast, females with MDD had increased sleep latency, slightly longer total sleep time, and increased \% stage 1 sleep $(\mathrm{p}<.01)$ compared to female controls. Also note that a completely different combination of sleep variables identified MDD in women compared to men. However, the regression model only accounted for $9 \%$ of the variance, significantly lower than that observed in men. See Table 3 for the means and standard deviations of these variables by group and sex.

\section{Sex Differences Within the MDD Group}

The last set of analyses focused exclusively on the MDD group, to establish which sleep variables best discriminated between males and females with MDD, and to determine the relationship between sleep and clinical features of depression.

Regression analyses were conducted with time in bed, total sleep time, sleep efficiency, sleep latency, \% stage 1, \% stage 2, \% slow wave, \% REM, arousals in the last 150 minutes of sleep, ascents to stage 1 per hour, REM density, and REM latency predicting sex. Although these variables 
Table 2. Means and Standard Deviations of Sleep Stage Variables by Groups

\begin{tabular}{|c|c|c|c|c|}
\hline & \multicolumn{2}{|c|}{$\begin{array}{l}\text { Healthy Controls } \\
\qquad n=62\end{array}$} & \multicolumn{2}{|c|}{$\begin{array}{c}\text { MDD } \\
n=129\end{array}$} \\
\hline & $\mathbf{M}$ & SD & $\mathbf{M}$ & SD \\
\hline Sleep latency $\dagger$ & 10.9 & 11.9 & 16.7 & 14.9 \\
\hline Time in bed & 433.9 & 40.6 & 447.24 & 43.4 \\
\hline Sleep efficiency & 92.3 & 4.3 & 89.8 & 6.9 \\
\hline$\%$ stage 1 & 14.6 & 6.4 & 13.5 & 6.7 \\
\hline$\%$ stage 2 & 54.6 & 7.7 & 55.2 & 8.5 \\
\hline$\%$ slow wave & 6.4 & 6.2 & 5.3 & 6.4 \\
\hline Arousals/ last 150 mins $\dagger$ & 9.4 & 3.8 & 8.0 & 4.2 \\
\hline Ascents to stage $1 /$ hour & 3.5 & 1.7 & 3.5 & 2.1 \\
\hline Stage changes/hour & 21.6 & 5.3 & 19.5 & 6.3 \\
\hline REM density $\dagger$ & 2.2 & 0.6 & 2.5 & 0.7 \\
\hline REM latency $\dagger$ & 67.3 & 15.8 & 70.5 & 22.9 \\
\hline
\end{tabular}

Note. $\mathrm{M}=$ mean; $\mathrm{SD}=$ standard deviation; $\mathrm{MDD}=$ major depressive disorder.

$\dagger$ Stepwise regression equation predicting group membership, $\mathrm{R}^{2}=.13, \mathrm{p}<.001$.

Table 3. Means and Standard Deviations of Selected Sleep Stage Variables by Group and Sex

\begin{tabular}{|c|c|c|c|c|c|c|c|c|}
\hline & \multicolumn{2}{|c|}{$\begin{array}{l}\text { Healthy Control Males } \\
\qquad n=32\end{array}$} & \multicolumn{2}{|c|}{$\begin{array}{l}\text { Males with MDD } \\
\qquad n=41\end{array}$} & \multicolumn{2}{|c|}{$\begin{array}{l}\text { Healthy Control Females } \\
\qquad n=30\end{array}$} & \multicolumn{2}{|c|}{$\begin{array}{l}\text { Females with MDD } \\
\qquad n=88\end{array}$} \\
\hline & M & SD & $\mathbf{M}$ & SD & $\mathbf{M}$ & SD & $\mathbf{M}$ & SD \\
\hline Sleep latencył & 9.8 & 14.1 & 15.3 & 16.9 & 12.3 & 9.0 & 17.3 & 13.9 \\
\hline Total sleep time & 395.8 & 35.9 & 401.6 & 44.9 & 405.5 & 38.7 & 409.5 & 47.7 \\
\hline Sleep efficiency $\dagger$ & 92.4 & 3.8 & 90.4 & 5.8 & 92.2 & 4.9 & 89.5 & 7.5 \\
\hline REM latency & 68.7 & 18.2 & 74.2 & 24.2 & 65.7 & 12.9 & 68.8 & 22.3 \\
\hline$\%$ stage $1 \ddagger$ & 14.9 & 6.1 & 15.9 & 7.9 & 14.3 & 6.8 & 12.4 & 5.7 \\
\hline$\%$ stage 2 & 54.9 & 7.6 & 53.9 & 8.3 & 54.3 & 7.9 & 55.8 & 8.6 \\
\hline$\%$ awake \& move & 5.3 & 2.6 & 6.4 & 5.4 & 4.6 & 3.3 & 6.1 & 5.1 \\
\hline Ascents to stage $1 /$ hour $\dagger$ & 3.4 & 1.4 & 4.1 & 2.3 & 3.5 & 1.9 & 3.4 & 1.9 \\
\hline Stage changes/hour $\dagger$ & 21.6 & 4.9 & 20.6 & 6.9 & 21.5 & 5.7 & 18.9 & 6.1 \\
\hline REM density $\dagger$ & 2.2 & 0.6 & 2.7 & 0.8 & 2.2 & 0.6 & 2.5 & 0.7 \\
\hline
\end{tabular}

Note. $\mathrm{M}=$ mean $\mathrm{SD}=$ standard deviation; $\mathrm{MDD}=$ sample with major depressive disorder

$\dagger$ Stepwise regression equation predicting group membership for males, $\mathrm{R}^{2}=.26, \mathrm{p}<.001$.

$¥$ Stepwise regression equation predicting group membership for females, $\mathrm{R}^{2}=.09, \mathrm{p}<.01$. 
together accounted for $17 \%$ of the variance $\left(\mathrm{r}^{2}=.17, \mathrm{p}=\right.$ $.05)$, only two of them significantly differentiated males with MDD from females with MDD, \% slow-wave $(\mathrm{p}=.04)$ and sleep efficiency $(p=.03)$. As shown in Table 3 , men with MDD had significantly less slow-wave sleep than women, but their sleep efficiency was higher. Entering the variables into the regression in a step-wise model, eliminating any redundant variables, \% Stage 1 sleep was the single best discriminator between males and females with MDD. Men with MDD had higher \% Stage 1 sleep than women $\left(r^{2}=.09, \mathrm{p}<\right.$ $.001)$.

The relationship among sleep and clinical variables was also assessed in men and women with MDD. Clinical variables considered were: number of previous episodes of MDD, age at onset of first episode, and current symptom severity (HRS-D score). Significantly different patterns emerged for men and women with MDD, with sleep variables accounting for significantly more of the variance in the course of illness measures in men than in women. Further, the sleep variables that were associated with clinical course of MDD also differed between the sexes. In men, sleep latency, time in bed, and stage changes per hour accounted for $25 \%$ of the variance in age of onset of MDD $(p=.02)$. An earlier age of onset was associated with longer sleep latency, shorter time in bed, and more stage changes per hour of sleep. In other words, more disturbed sleep was found in those men with an earlier age of onset of the first episode of MDD. For women, total sleep time and sleep latency accounted for $13 \%$ of the variance in age at onset of MDD ( $p=$ .003 ), half that of MDD men. An earlier age of onset of the first episode of MDD was associated with less total sleep time but shorter sleep latency in women.

With regard to symptom severity, sleep latency and \% REM accounted for $25 \%$ of the variance in HRS-D score in males $(\mathrm{p}=.008)$. More severe depressive symptoms were associated with longer sleep latency and more REM sleep in men. By contrast, HRS-D symptom severity related to sleep efficiency in females $(\mathrm{p}=.05)$ but accounted for a small portion of the variance (4\%). More severe depressive symptoms were associated with lower sleep efficiency in women.

Number of previous episodes of MDD was also significantly related to sleep measures, but only among women. Sleep stability variables (\% awake and movement, stage changes per hour, and sleep latency) accounted for $9 \%$ of the variance in number of episodes of MDD $(p=.005)$. More lifetime episodes of depression was associated with longer sleep latency, a higher percentage of time spent awake and more stage changes per hour. In other words, sleep was more disturbed in those women with more lifetime episodes of MDD. Number of episodes of MDD was not related to sleep measures in men.

No further analyses were conducted.

\section{DISCUSSION}

A combination of sleep variables emerged to best discriminate between this large sample of never-depressed healthy controls and individuals with MDD. These variables included sleep latency, REM density, REM latency, and arousals in the last 150 minutes of sleep. Consistent with previous findings regarding sleep in MDD, our MDD group took longer to fall asleep $[17,38,39]$ and had more phasic
REM, reflected in increased REM density [27, 39], compared to HCs. However, those with MDD had longer REM latency than the $\mathrm{HC}$ group, a result that we have reported previously [30, 32, 33], but is inconsistent with other published work [27]. Moreover, several variables that are commonly thought to be associated with MDD, including sleep efficiency $[27,38,40,41]$ and percent slow-wave sleep [38, 39] were not significantly different between $\mathrm{HC}$ and participants with MDD.

In the present study, every effort was made to control for potential confounding variables. Participants were required to maintain a regularized sleep-wake schedule, minimize caffeine usage ( 1 cup coffee equivalent per day prior to 11 am), and refrain from napping or from drug and alcohol use prior to the study. They were also given ample time to adapt to the sleep environment by taking an extensive laboratory tour accompanied by the research assistant who would later conduct their sleep study. We also screened for independent sleep disorders using a conservative exclusion for periodic limb movements or apneas and hypopneas, using a criterion of 5 events per hr. In addition, we matched the age of the $\mathrm{HC}$ and MDD and controlled for any residual age effects statistically.

Participants were carefully screened for inclusion in the study. Those with depression were otherwise medically healthy, free of comorbid Axis I diagnoses, and not using pharmacological treatments for their depression. Although this increases the internal validity of the study, it limits the generalizability of the results, particularly to individuals with depression who have medical or psychiatric comorbidities.

The end result of these procedures is likely to have reduced sleep differences between patients and controls and resulted in the exclusion of more severely depressed patients. While we maintained higher statistical control by eliminating potential confounds, this is a limitation to the present study and interpretation of findings. Nevertheless, we and others have suggested that no single sleep measure reliably differentiates depressed patients from healthy individuals $[25,31]$ and the findings of the present study indicate relatively small differences between MDD and HC groups.

However, the distinction between patients and controls is moderated by sex. Sleep variables accounted for more than one-quarter of the variance in differentiating men with MDD from HCs. Men with MDD had lower sleep efficiency and higher REM density. By contrast, women with MDD took longer to fall asleep and had more light Stage 1 sleep than $\mathrm{HC}$ females, but the overall regression accounted for less than $10 \%$ of the variance in predicting group membership in women. Thus, different sleep variables differentiated MDD men from HC subjects than that obtained in women. Stated differently, the sleep of MDD women was closer to $\mathrm{HC}$ women. Our own research group has suggested that there is greater vulnerability to MDD in women than in men and that sleep EEG measures may reflect this increased vulnerability [30]. Thus, one might expect sleep measures to differ less in women than in men. However, mean differences were not always smaller between MDD and HC women and thus it is not clear whether these findings relate specifically to risk for MDD. 
Nevertheless, sex differences were evident within the MDD group. Men with MDD had significantly less slowwave sleep and more Stage 1 sleep than in MDD women. These findings are in general agreement with previous research $[26,27,29]$. It should be noted, however, that there are only a handful of studies on sex differences in sleep in depression.

We also found significant sex differences in which sleep variables predicted age at onset of MDD, severity of depressive symptoms, and number of episodes of depression. Moreover, the relationship between clinical and sleep measures was stronger in men than in women. An earlier age of onset was significantly associated with longer sleep latency, shorter time spent in bed, and more stage changes per hour for males. Thus, more sleep disturbance was seen in the men who had spent more time ill with depression. In contrast, women who spent more time ill with depression slept less, but took a shorter time to fall asleep, and these variables accounted for roughly half the variance than that obtained in regressions analysis in the men. More severe depressive symptoms were associated with taking a longer time to fall asleep and more REM sleep in men with these two variables accounting for nearly a quarter of the variance in depressive symptoms. By sharp contrast, only sleep efficiency related to depression severity in MDD females, and it only accounted for $4 \%$ of the variance. Women with more severe depressive symptoms had lower sleep efficiency, as might be expected, but the relationship among clinical features of depression and sleep measures was significantly weaker than that observed in men. However, one clinical course variable did bear a stronger relationship to sleep measures in women than in men, the number of previous episodes of depression. Those women with more lifetime episodes of depression took longer to fall asleep, had more stage changes per hour, and spent more time awake during the night. None of the sleep measures predicted number of depressive episodes in men.

It is reasonable to expect sleep to be more disturbed in those with a worse clinical course of depression. Spending more years ill with depression, having greater symptom severity and experiencing more episodes of depression could result in more disturbed sleep. A recent study does support more disturbed sleep in more severe depressions, but did not assess sex differences [42]. However, a PubMed search revealed no published reports evaluating sex differences in the relationship between sleep and clinical variables. We did find one study of the relationship of symptom severity and sleep, exclusively in depressed men. Unlike the results obtained in the present study, Hubain et al. [43] reported no correlation between REM sleep measures and severity of depression. Thus, it is a little difficult to place the findings of the present study in the context of previous work. Further, a replication would increase confidence in our conclusions. A prospective longitudinal assessment of how sleep changes with course of illness within an individual would be the ideal study design. Nevertheless, this study does highlight the importance of considering sex as a moderating variable.

\section{ACKNOWLEDGEMENTS}

This study was supported by grant RO1-MH61515 from the National Institute of Mental Health.

\section{REFERENCES}

[1] Kessler RC, Berglund P, Demler O, et al. The epidemiology of major depressive disorder: results from the National Comorbidity Survey Replication (NCS-R). JAMA 2003; 289: 3095-105.

[2] Kessler RC. Prevalence, severity, and comorbidity of 12-month DSM-IV disorders in the National Comorbidity Survey Replication. Arch Gen Psychiatry 2005; 62: 617- 27.

[3] Kessler RC, Borges G, Walters EE. Prevalence of and risk factors for lifetime suicide attempts in the National Comorbidity Survey. Arch Gen Psychiatry 1999; 56: 617-26.

[4] Hirschfeld RM, Montgomery SA, Keller MB, et al. Social functioning in depression: a review. J Clin Psychiatry 2000; 61: 268-75.

[5] Spitzer RL, Kroenke K, Linzer M, et al. Health-related quality of life in primary care patients with mental disorders: Results from the PRIME-MD 1000 study. JAMA 1995; 274: 1511-7.

[6] Wells KB, Stewart A, Hays RD, et al. The functioning and wellbeing of depressed patients: Results from the medical outcomes study. JAMA 1989; 262: 914-9.

[7] American Psychiatric Association: Diagnostic and Statistical Manual of Mental Disorders, 4th, text revision ed. Washington, D.C.: American Psychiatric Press 2000.

[8] Breslau N, Roth T, Rosenthal L, Andreski P. Sleep disturbance and psychiatric disorders: A longitudinal epidemiological study of young adults. Biol Psychiatry 1996; 39: 411-8.

[9] Ford DE, Kamerow DB. Epidemiologic study of sleep disturbances and psychiatric disorders: An opportunity for prevention? JAMA 1989; 262: 1479-84.

[10] Buysse DJ. Prevalence, course, and comorbidity of insomnia and depression in young adults. Sleep 2008; 31: 473-80.

[11] Armitage R. The effects of antidepressants on sleep in patients with depression. Can J Psychiatry 2000; 45: 803-9.

[12] Thase ME. Depression, sleep, and antidepressants. J Clin Psychiatry 1998; 59(Suppl 4): 55-65.

[13] Thase ME, Buysse DJ, Frank E, et al. Which depressed patients will respond to interpersonal psychotherapy? The role of abnormal EEG sleep profiles. Am J Psychiatry 1997; 154: 502-9.

[14] Bolton JM. Exploring the correlates of suicide attempts among individuals with major depressive disorder: findings from the national epidemiologic survey on alcohol and related conditions. J Clin Psychiatry 2008; 69: 1139-49.

[15] Wingard DL. Mortality risk associated with sleeping patterns among adults. Sleep 1983; 6: 102-7.

[16] Fawcett J. Time-related predictors of suicide in major affective disorder. Am J Psychiatry 1990; 147: 1189-94.

[17] Goetz RR. EEG sleep of young adults with major depression: a controlled study. J Affect Disord 1991; 22: 91-100.

[18] Armitage R, Trivedi M, Hoffmann R, Rush AJ. Relationship between objective and subjective sleep measures in depressed patients and healthy controls. Depress Anxiety 1997; 5: 97-102.

[19] Perlis ML, Giles DE, Buysse DJ, Thase ME, Tu X, Kupfer DJ. Which depressive symptoms are related to which sleep electroencephalographic variables? Biol Psychiatry 1997; 42: 904-13.

[20] Lauer CJ, Riemann D, Wiegand M, Berger M. From early to late adulthood: Changes in EEG sleep of depressed patients and healthy volunteers. Biol Psychiatry 1991; 29: 979-93.

[21] Kupfer DJ, Reynolds CF 3rd, Ulrich RF, Grochocinski VJ. Comparison of automated REM and slow-wave sleep analysis in young and middle-aged depressed subjects. Biol Psychiatry 1986; 21: 189-200.

[22] Akiskal HS, Lemmi H, Dickson H, King D, Yerevanian B, Van Valkenburg C. Chronic depressions. Part 2: Sleep EEG differentiation of primary dysthymic disorders from anxious depressions. J Affect Disord 1984; 6: 287-95.

[23] Benca RM. Sleep in psychiatric disorders. Neurol Clin 1996; 14: 739-64.

[24] Armitage R, Hoffmann RF. Sleep EEG, depression and gender. Sleep Med Rev 2001; 5: 237-46.

[25] Benca RM, Obermeyer WH, Thisted RA, Gillin JC. Sleep and psychiatric disorders. A meta-analysis. Arch Gen Psychiatry 1992; 49: 651-68.

[26] Reynolds CF, Kupfer DJ, Thase ME, et al. Sleep, gender, and depression: An analysis of gender effects on the electroencephalographic sleep of 302 depressed outpatients. Biol Psychiatry 1990; 28: 673-84.

[27] Riemann D. Sleep in depression: The influence of age, gender and diagnostic subtype on baseline sleep and the cholinergic REM in- 
duction test with RS 86. Eur Arch Psychiatry Clin Neurosci 1994; 243: 279-90.

[28] Armitage R. Microarchitectural findings in sleep EEG in depression: Diagnostic implications. Biol Psychiatry 1995; 37: 72-84.

[29] Armitage R, Hoffmann R, Fitch T, Trivedi M, Rush AJ. Temporal characteristics of delta activity during NREM sleep in depressed outpatients and healthy adults: Group and sex effects. Sleep 2000; 23: 607-17.

[30] Armitage R. Sleep and circadian rhythms in mood disorders. Acta Psychiatr Scand Suppl 2007; 433: 104-15.

[31] Armitage R, Hoffmann R, Trivedi M, Rush AJ. Slow-wave activity in NREM sleep: Sex and age effects in depressed outpatients and healthy controls. Psychiatry Res 2000; 95: 201-13.

[32] Armitage R, Hoffmann RF, Rush AJ. Biological rhythm disturbance in depression: Temporal coherence of ultradian sleep EEG rhythms. Psychol Med 1999; 29: 1435-48.

[33] Spitzer RL, Williams JB, Gibbons M. The Structured Clinical Interview for DSM-III-R (SCID). New York State Psychiatric Institute: New York 1986.

[34] Hamilton M. A rating scale for depression. J Neurol Neurosurg Psychiatry 1960; 23: 56-62.

[35] Rechtstaffen A, Kales A. A Manual of Standardized Terminology, Techniques, and Scoring Systems for Sleep Stages of Human Subjects. Washington, D.C.: U.S. Government Printing Office 1968.
[36] Armitage R, Rush AJ, Trivedi M, Cain J, Roffwarg HP. The effects of nefazodone on sleep architecture in depression. Neuropsychopharmacology 1994; 10: 123-7.

[37] Kerlinger FN, Pedhazur EJ. Multiple Regression in Behavioral Research. New York, NY: Holt, Rinehart and Winstone, Inc., 1973.

[38] Mendlewicz J. Sleep electroencephalography in depressive illness. A collaborative study by the World Health Organization. Br J Psychiatry 1991; 159: 505-9.

[39] Debus JR, Rush AJ. In: McCann CD, Endler NS, Eds. Depression: New Directions in Theory, Research, and Practice. Toronto, Wall \& Emerson, Inc. 1990; 337-60.

[40] Arriaga F. The sleep of dysthymic patients: a comparison with normal controls. Biol Psychiatry 1990; 27: 649-56.

[41] Thase ME. Electroencephalographic sleep profiles in singleepisode and recurrent unipolar forms of major depression: I. Comparison during acute depressive states. Biol Psychiatry 1995; 38: 506-15.

[42] Gupta R, Dahiya S, Bhatia M. Effect of depression on sleep: Qualitative or quantitative? Indian J Psychiatry 2009; 51: 117-21.

[43] Hubain P, LeBon O, Vandenhende F, Van Wijendaele, Linkowski P. Major depression in males: Effects of age, severity and adaptation on sleep variables. Psychiatry Res 2006; 145: 169-77.

(C) Swanson et al.; Licensee Bentham Open.

This is an open access article licensed under the terms of the Creative Commons Attribution Non-Commercial License (http://creativecommons.org/licenses/by-nc/3.0/) which permits unrestricted, non-commercial use, distribution and reproduction in any medium, provided the work is properly cited. 